\title{
Impaction caused by a Rare erupted peripheral compound Odontoma
}

\author{
anand marya ${ }^{1}$ and Adith Venugopal ${ }^{2}$ \\ ${ }^{1}$ University of Puthisastra \\ ${ }^{2}$ Saveetha University Saveetha Dental College
}

November 8, 2021

\begin{abstract}
Odontomas are common occurrences in the oral cavity and can be classified as complex or compound. Erupted peripheral compound odontomas are rare and are present in the extra-osseous soft tissues. In this case the odontoma led to the impaction of permanent teeth for which removal of the lesion was advised.
\end{abstract}

\section{Introduction}

Odontomas are common odontogenic tumors and are classified into compound or complex tumors ${ }^{1}$. In this case, a 17-year-old boy reported to the university clinic with a problem of missing teeth. On intraoral examination, the patient had a missing left lateral incisor and canine, and 'Denticles' were present on the gingiva in the affected $\operatorname{area}^{2}$ (Figure1). The patient was asymptomatic on palpation, and the denticles demonstrated biofilm and gingival inflammation around them. Radiographic analysis demonstrated the presence of various irregular tooth structures made up of a crown and the root without bony involvement (Figure 2). The permanent left maxillary lateral incisor and canine were impacted due to their eruptive path being obstructed. Based on these findings, the case was diagnosed as that of Peripheral compound Odontoma. Since the patient was looking for orthodontic treatment, the patient was advised to remove the Odontoma and undergo fixed appliance therapy. Radiologic examination after the alignment of the affected teeth revealed that the lateral incisor root was dilacerated, requiring careful management after that (Figure 3). Dentists must be aware of such problems as these, if not removed, will gradually increase in size and lead to other problems such as adjacent tooth mobility, periodontal destruction, and even esthetic concerns.

Figure 1: Clinical intra-oral presentation of the erupted peripheral compound odontoma 

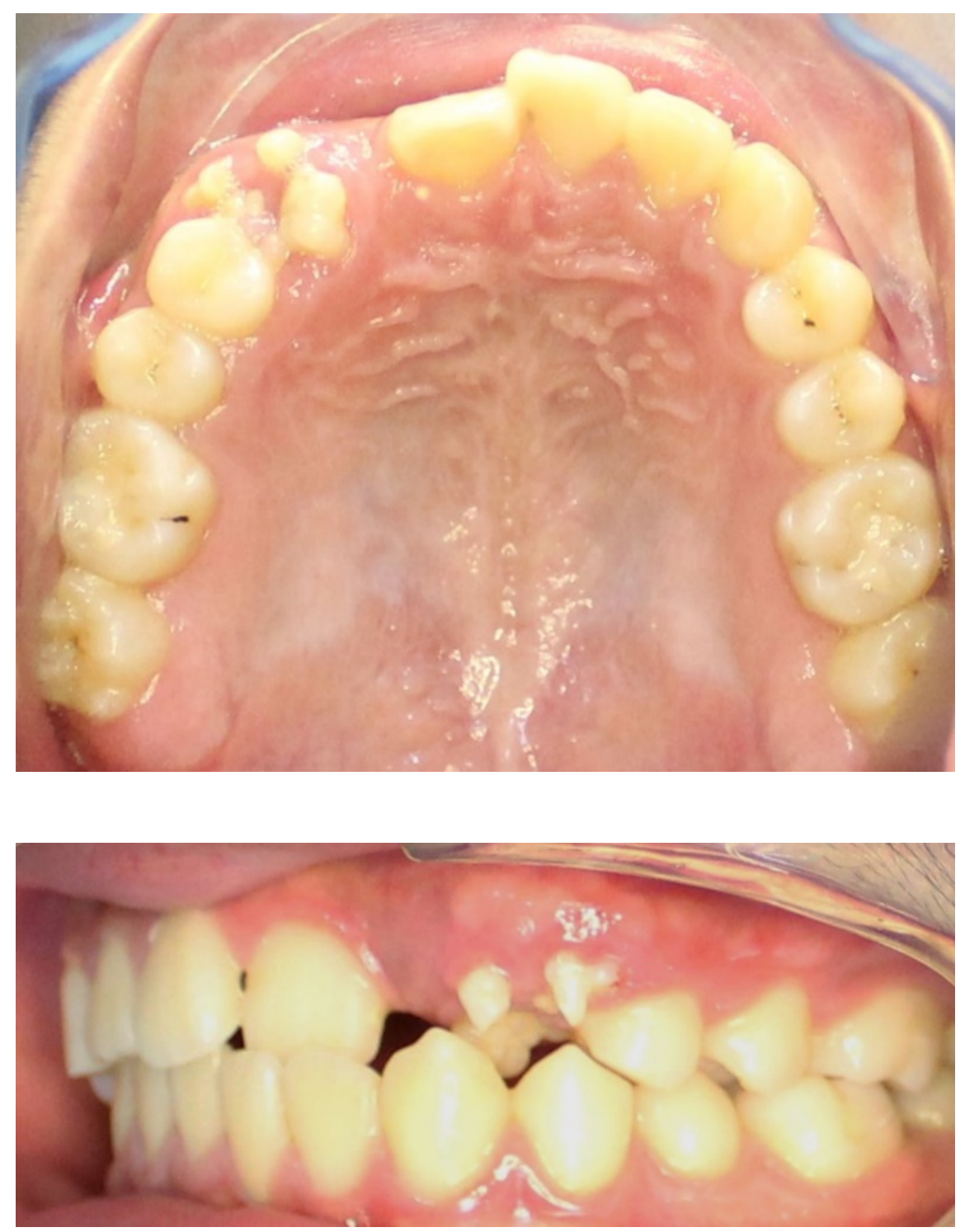

Figure 2 : Pre-treatment panoramic image of the Peripheral Compound Odontoma 

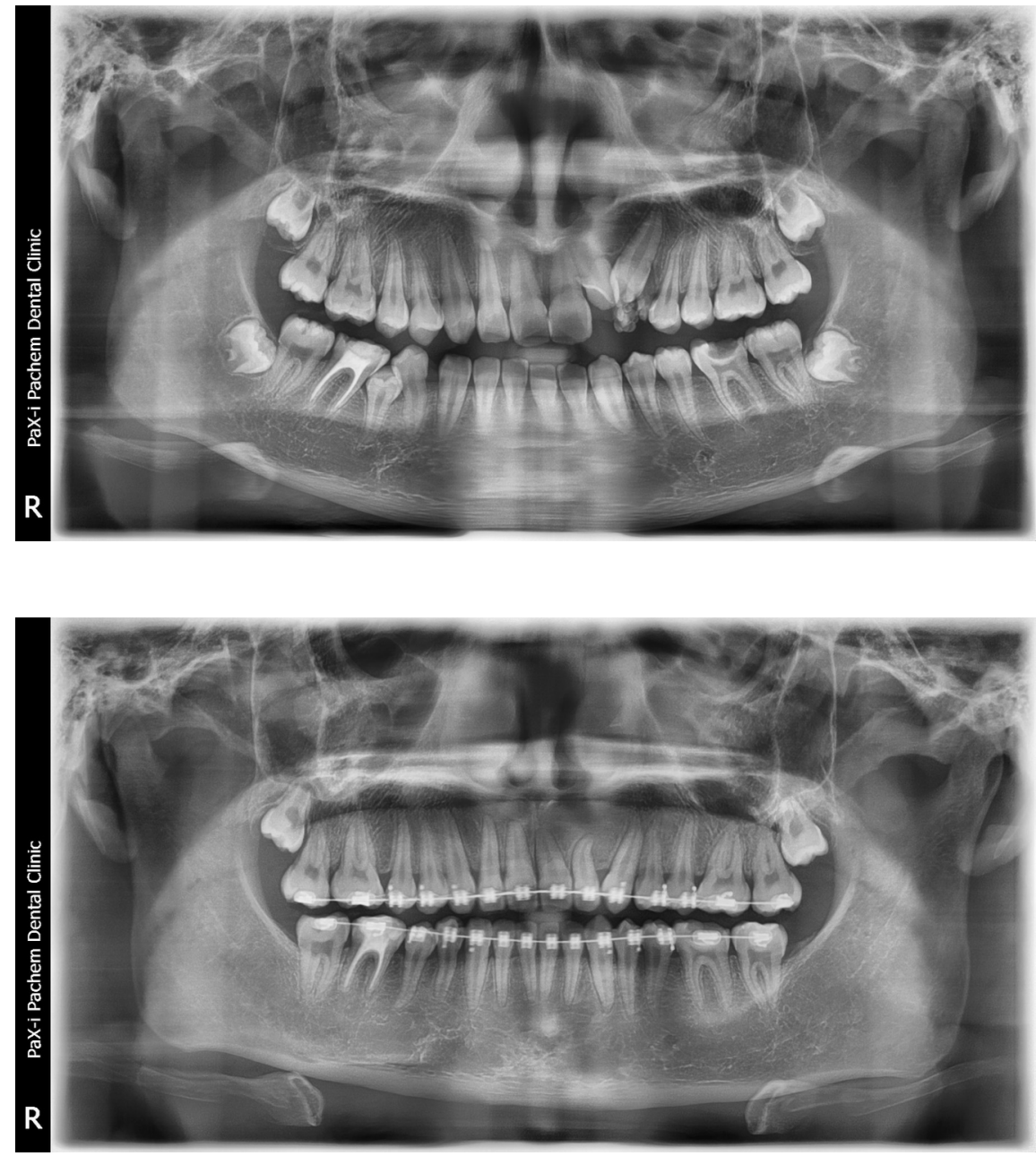

Figure 3: Post-removal panoramic image of the Odontoma with the subsequent alignment of the impacted teeth.

\section{Ethical Statement}

Because this report involves no experiment, ethics approval is waived.

\section{Acknowledgment}

The authors would like to thank the patient for giving consent.

\section{Patient consent}

Written informed consent was obtained from the patient to publish this report in accordance with the journal's patient consent policy

\section{Conflict of Interest}

The authors made no disclosures.

Author contributions: AM: patient treatment AM \& AV: diagnosis and treatment planning AM: manuscript preparation, AM \& AV: review and editing

\section{References}


Oliveira BH, Campos V, Marçal S: Compound odontoma- diagnosis and treatment: three case reports. Oral Pathology. 2001, 23: 151-157.

Hanemann, J.A., Oliveira, D.T., Garcia, N.G. et al. Peripheral compound odontoma erupting in the gingiva. Head Face Med 9, 15 (2013). 